\title{
Esquema de integração de hipermídias adaptativas em ambientes virtuais de aprendizagem
}

\author{
Erika Handa Nozawa ${ }^{1}$, Elaine Harada Teixeira de Oliveira ${ }^{2}$, Rosa Maria Vicari ${ }^{3}$, \\ Alberto Nogueira de Castro Júnior ${ }^{2}$ \\ ${ }^{1}$ Programa de Pós-Graduação em Informática - Universidade Federal do Amazonas \\ (UFAM) - Manaus - AM - Brazil \\ ${ }^{2}$ Departamento de Ciência da Computação - Universidade Federal do Amazonas \\ (UFAM) - Manaus - AM - Brazil \\ ${ }^{3}$ Instituto de Informática - Universidade Federal do Rio Grande do Sul (UFRGS) - \\ Porto Alegre - RS - Brazil \\ erika.handalgmail.com, elaine@dcc.ufam.edu.br, rosa@inf.ufrgs.br, \\ albertodufam.edu.br
}

Resumo. No processo de ensino-aprendizagem, muitos fatores são importantes. Este trabalho aborda a questão de que a manutenção da atenção é fator primordial e tenta fazê-lo através do princípio da relevância. Neste caso, o sistema proposto utiliza uma ontologia de domínio da língua japonesa para tentar apresentar opções de aprendizado personalizado e direcionado ao contexto, através de um agente pedagógico animado. Este artigo apresenta a integração dos conceitos de ontologia, Teoria da Relevância e agentes pedagógicos para a concepção de um ambiente hipermídia para auxiliar no processo de aprendizagem.

Palavras-chave: hipermídia adaptativa, ontologia, Teoria da Relevância, agentes pedagógicos.

\section{Schema of integration of adaptive hypermedia in virtual learning environments}

\begin{abstract}
Many factors are important in the learning process. This work addresses the issue that the maintenance of attention is an important factor and tries to do so through the principle of relevance. In this case, the proposed system uses ontology of domain of the Japanese language to try to provide options for personalized learning oriented to the context, through an animated pedagogical agent. This paper presents the integration of the concepts of ontology, the Relevance Theory and pedagogical agents to design a hypermedia environment to assist in the learning process.
\end{abstract}

Keywords: adaptive hypermedia, ontology, Relevance Theory, pedagogical agents.

\section{Introdução}

A educação a distância tem realizado um papel importante na evolução dos programas de educação e treinamento, os quais vêm sendo cada vez mais procurados em vista da 
crescente competitividade do mercado de trabalho mundial. O potencial da Internet tem possibilitado uma forma de aprendizado a distância, onde as informações são distribuídas em formatos e localização diversos. A realidade atual nos permite utilizar ambientes de ensino-aprendizagem tanto presenciais como virtuais. Uma das constantes, que ocorre tanto no espaço presencial quanto no virtual, é a diversidade de alunos, nos mais variados aspectos.

O ambiente presencial conta com a vantagem da possibilidade de convivência com o aluno. Para ele, é possível ter uma noção do perfil de aprendizagem dos alunos e das dificuldades de aprendizagem que porventura possam ter. Ele conta ainda, com a possibilidade de uma observação direta do comportamento dos alunos. Com toda essa percepção, ele pode adotar estratégias que beneficiem o grupo como um todo.

Já no ambiente virtual, a mesma situação não ocorre. Conforme Gasparini (2002), um dos problemas comuns nos Ambientes Virtuais de Aprendizagem (AVA) é que a maioria se limita a uma rede de páginas de hiperdocumentos estáticos e que não aproveitam todas as funcionalidades e características das aplicações hipermídia. Outro problema é que os estudantes são tratados como se tivessem sempre o mesmo perfil, metas e conhecimentos. Para o professor fica difícil perceber os alunos, tanto em termos de presença, de atenção e de dedicação. Muitas vezes, o retorno que ele tem não é tão fiel à realidade. $\mathrm{O}$ aluno, por outro lado pode se sentir desconfortável. Ele não desfruta do ambiente coletivo, onde há troca de experiências e convívio. Além disso, ele pode se sentir frustrado por não ter um curso personalizado de acordo com suas preferências, necessidades, conhecimentos, objetivos e estilo.

Para isso, seria necessário considerar os processos cognitivos e mentais do aluno, algo muito difícil de ser feito. Uma das funções cognitivas de interesse deste trabalho é a atenção. Wiliam James citado por Brown (2007) escreveu há mais de cem anos que a atenção é "a mente tomando posse, de forma vívida e clara, de um entre aqueles que parecem ser outros possíveis objetos de uma seqüência de pensamento.[...] Isso implica exigir a remoção da atenção em algumas coisas, para lidar eficientemente com outras."

Acredita-se que o nível de atenção que um indivíduo aplica a um determinado assunto ou suposição apresentada está totalmente relacionado com grau de relevância que a mesma considera para a sua representação de mundo. Com isso em vista, são bastante pertinentes as considerações da Teoria da Relevância (Sperber e Wilson, 1995) que estabelece relações entre suposição, contexto e atenção. Para alguma suposição ser relevante num contexto, é necessário que surjam conexões entre as novas suposições e aquelas já existentes, ou seja, que já fazem parte do contexto.

O objetivo do trabalho é propor um mecanismo de apoio ao processo de ensinoaprendizagem que leve em consideração os processos mentais e cognitivos do aluno, mais especificamente os relacionados à manutenção da atenção. A proposta é modelar o domínio de preparação para um teste proficiência da língua da japonesa levando-se em consideração as várias possibilidades de contexto. Com essa modelagem em mãos, pretende-se aplicar, através de um agente de software inteligente e afetivo, estratégias pedagógicas e afetivas, na manutenção da atenção através da tentativa de obtenção de relevância ótima, de acordo com os objetivos propostos pelo aluno.

O escopo deste trabalho é apresentar uma proposta de esquema que transforma um AVA em um ambiente hipermídia adaptativo, acompanhada das primeiras etapas da 
pesquisa. Para tal, será apresentado na próxima seção o esquema de integração a partir do eJLPT. Em seguida, uma seção sobre a Teoria da Relevância, com os conceitos pertinentes a esse trabalho. Na seção 4, é feita uma exposição sobre ontologias e suas possibilidades de uso neste trabalho. A seção 5 apresenta, brevemente, o agente inteligente a ser utilizado. Para concluir, são apresentadas as considerações finais.

\section{Esquema de integração a partir do eJLPT}

O simulador eJLPT (2005) é uma ferramenta hipermídia de apoio educacional para alunos com o objetivo de exercitar seus conhecimentos na língua japonesa, através de simulações do JLPT, o exame de certificação em proficiência da língua japonesa. As funcionalidades do ambiente eJLPT consistem dos módulos Professor e Aluno, onde o primeiro disponibiliza as aulas com a ementa e formula os exercícios, e o aluno, por sua vez, possui o ambiente para navegar nas aulas e exercitar seus conhecimentos. Uma tela do sistema eJLPT pode ser visualizada na Figura 1.

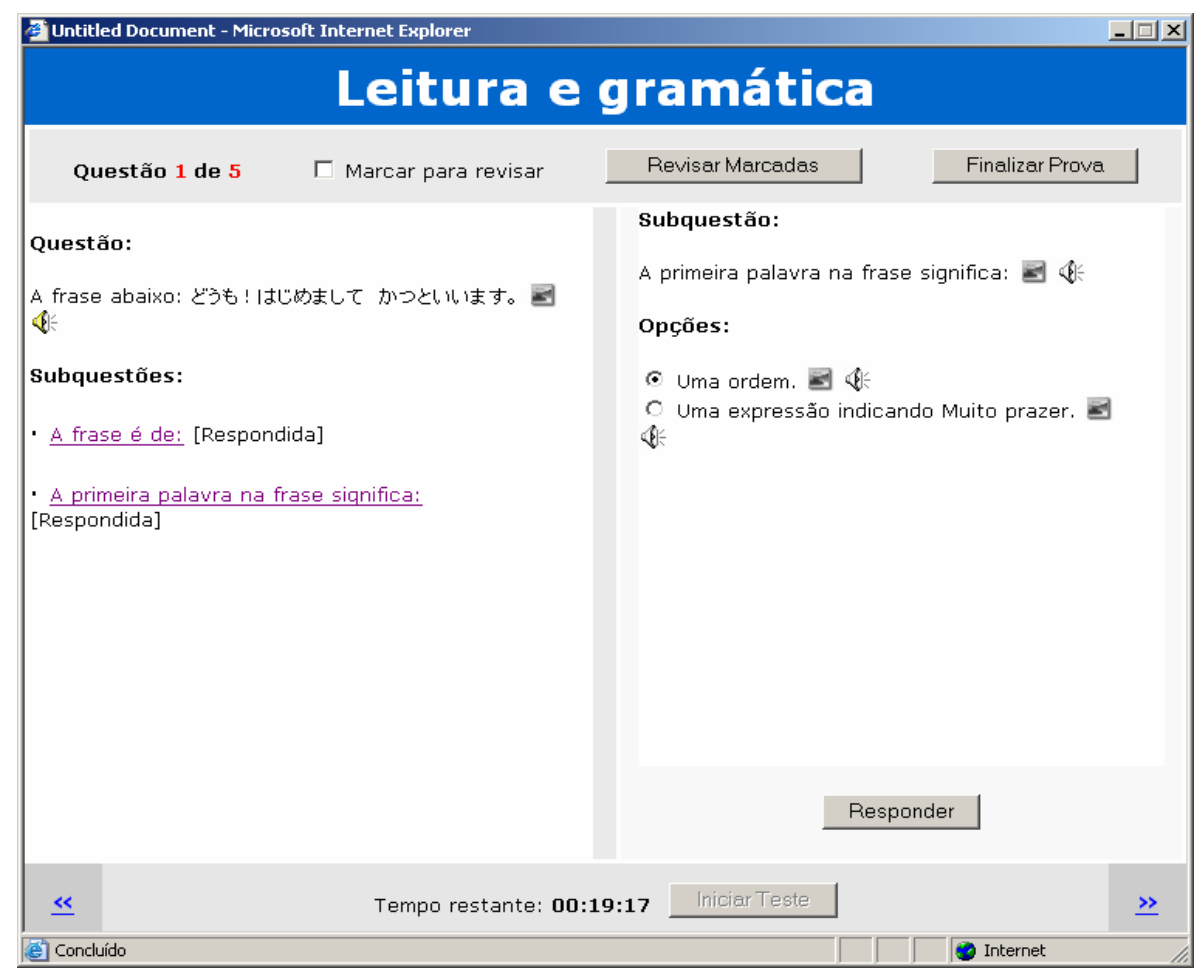

Figura 1. Ambiente do aluno - Tela da prova em curso do sistema eJLPT

Pôde-se perceber, no decorrer do uso do eJLPT, a necessidade de criação de cenários automáticos para atender algumas expectativas dos alunos e dos professores:

1. Como está minha evolução na aprendizagem para a aprovação no JLPT? Tenho parâmetros automáticos para "medir" o meu aprendizado no contexto do JLPT?

2. Quais os recursos disponibilizados pelo sistema para adaptar minhas necessidades de aprendizagem ao contexto do JLPT?

3. O professor pode me guiar e direcionar melhor para que eu não empenhe esforços desnecessários em determinados assuntos que não são importantes ao JLPT? Há ferramentas neste contexto para o professor? E para o aluno?

A partir das solicitações acima, foi possível elicitar requisitos para uma proposta de arquitetura onde as ontologias pudessem mapear tais visões. $\mathrm{O}$ primeiro esquema proposto, na Figura 2a, sugere que para se integrar hipermídias adaptativas em AVA, 
faz-se necessário a escolha de um modelo de adaptação que sustente um modelo do usuário definido. A partir das necessidades de adaptação mapeadas do usuário, associase um mecanismo de adaptação capaz de implementá-lo. O esquema seguinte, visualizado na Figura $2 b$, é a sugestão de instanciação proposta neste trabalho para a validação, denominada de Poliglota.

Uma forma de validação (funcional) do esquema proposto é a análise dos resultados através de uma possível instanciação do mesmo. Assim, se a integração dos elementos instanciados é suficiente para se obter uma hipermídia adaptativa nos contextos funcionais elicitados, a validação do esquema foi obtida.

A sugestão de instanciação do esquema proposto, o Poliglota, é a integração no AVA eJLPT, do modelo de adaptação associado ao modelo de usuário mapeado através de ontologias envolvendo a Teoria da Relevância e utilizando-se de um mecanismo de adaptação inteligente e a agente PAT, um agente pedagógico animado.

Acredita-se que os elementos adotados para a instanciação sugerida são factíveis de integração, pois cada elemento ora proposto é uma solução funcional existente e independente. As seções seguintes descrevem os elementos instanciados envolvidos e suas relações que permitem contextualizar a visão de integração em hipermídia adaptativa.

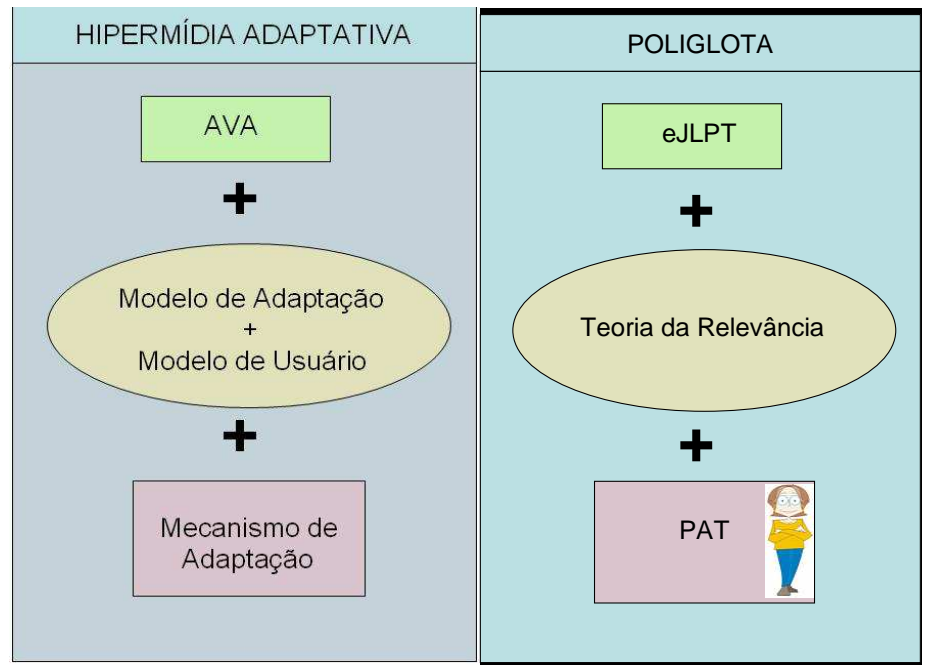

(a)

(b)

Figura 2 - Esquema de integração de hipermídias adaptativas em ambientes virtuais de aprendizagem e uma possível instanciação do esquema

\section{Teoria da Relevância}

Uma comunicação eficiente, tanto oral quanto escrita, é imprescindível para um processo de ensino-aprendizagem bem sucedido. Esse processo de comunicação pode ocorrer de várias formas, sendo de interesse deste trabalho, as formas em que se faz necessário um processo de reconhecimento ostensivo-inferencial para o devido entendimento de certo enunciado. Ou seja, deve haver o desejo de realmente ocorrer a comunicação através de estímulos explícitos e para tal deve haver a análise de tais estímulos e a conclusão de que o comunicador tem suas intenções ao fazê-lo.

Acredita-se que a interpretação de um enunciado parte de um conjunto de premissas, que geralmente é chamado de 'contexto'. "Um contexto é um construto 
psicológico, um subconjunto de suposições do ouvinte sobre o mundo." (Sperber e Wilson, 1995). São essas suposições, e não o estado atual do mundo, que afetam a interpretação de um enunciado.

É possível melhorar ou modificar um dado contexto. Não há modificação no contexto quando a informação é totalmente duplicada ou quando ela é não é relacionada com nenhuma informação antiga. Deve haver interação entre uma informação nova e uma antiga. "O contexto usado para processar novas suposições é um subconjunto de suposições antigas de um indivíduo, com o quais novas suposições se combinam para gerar uma variedade de efeitos contextuais" (Sperber e Wilson, 1995).

Os efeitos contextuais podem ocorrer, quando algumas suposições são apagadas do contexto; a força de algumas suposições do contexto é modificada, ou; há derivação de implicações contextuais, ou seja, há uma melhora na representação de mundo de um indivíduo.

Para haver relevância e, portanto, captar a atenção, deve haver efeitos contextuais. Quanto maiores os efeitos contextuais gerados por uma nova informação, maior a relevância da mesma. Efeitos contextuais é condição necessária e suficiente para relevância.

Cabe aqui, então uma definição classificatória do conceito de relevância (Sperber e Wilson, 1995): "Uma suposição é relevante em um contexto se e somente se ela tem um efeito contextual naquele contexto."

Para que haja relevância, algumas condições devem ser atendidas, o que nos leva uma definição comparativa:

1. Uma suposição é relevante em um contexto na medida em que seus efeitos contextuais nesse contexto são grandes.

2. Uma suposição é relevante em um contexto na medida em que o esforço requerido para processá-la nesse contexto é pequeno.

Além dessa relação entre relevância, efeito contextual e esforço processual, há de se levar em conta que há graus de relevância que variam conforme o efeito contextual e esforço processual. E deve-se considerar também que a relevância de uma suposição para um indivíduo pode ser um conceito tanto classificatório quanto comparativo.

Num processo de comunicação ostensivo-inferencial, o comunicador produz um estímulo que torna manifesto tanto ao comunicador quanto à audiência, que o comunicador pretende, através de estímulos, tornar manifesto à audiência um conjunto de suposições.

Para ser eficaz, um ato de comunicação ostensiva deve atrair a atenção da audiência. Nesse caso, um ato de ostensão é uma requisição à atenção. Pessoas prestam atenção a um fenômeno somente se ele lhe parece relevante.

O nível de relevância a ser considerado leva em conta o interesse tanto do comunicador quanto da audiência. Quando esse nível atinge seu ponto máximo, dá-se o nome de relevância ótima.

Considerando que há um comunicador, que pode estar se manifestando de forma oral ou escrita, e que este pretende tornar manifesto um conjunto de suposições ao endereçado, pela presunção da relevância ótima, acredita-se que o conjunto de suposições é relevante o suficiente para fazê-lo valer ao endereçado enquanto este processa estímulos ostensivos. Além disso, acredita-se que o estímulo ostensivo é o 
mais relevante que o comunicador poderia ter usado para comunicar o conjunto de suposições desejado.

Eis então o princípio de relevância, que irá nortear boa parte do trabalho apresentado: "Cada ato de comunicação ostensiva comunica a presunção de sua própria relevância ótima" (Sperber e Wilson, 1995).

Com base em todas as considerações apresentadas anteriormente, surgem algumas questões interessantes:

- Qual procedimento poderia ser usado para comparar os efeitos contextuais resultantes?

- Qual procedimento poderia ser usado para comparar o esforço necessário para processar uma suposição, tanto isolada de um contexto quanto em um contexto selecionado?

- Como atingir o nível de relevância ótima para um dado conjunto de suposições que se pretende apresentar?

Para essas questões, uma das soluções vislumbradas é a apresentada neste artigo. Acredita-se que o uso de ontologia pode favorecer a comparação dos efeitos contextuais resultantes, a comparação do esforço necessário para processar uma suposição e a obtenção do nível de relevância ótima.

\section{Ontologias}

Segundo Grubber (1993), "uma ontologia é uma especificação explícita de uma conceitualização." Conceitualização é um modelo abstrato do mundo que se quer representar e essa representação tem de ser explícita com especificação dos conceitos, propriedades e relações.

Borst (1991) modificou essa definição ao acrescentar que a conceitualização deve permitir o seu compartilhamento. Enfatizou que o conhecimento expresso deve ser do senso comum e não particular a quem está escrevendo e/ou expressando.

O processo de conceituação implica em definir um corpo de conhecimento, representado formalmente, que seja baseado nos seguintes elementos: objetos, entidades, relações entre objetos e entre conceitos. Formalmente, para especificar uma conceituação são necessários axiomas lógicos que restringem as possíveis interpretações dos termos definidos. Pragmaticamente, uma ontologia comum define o vocabulário com o qual perguntas e respostas serão trocadas entre agentes.

Para construir uma ontologia, é necessário decidir o seu tipo, atentar-se aos critérios de construção, usar uma metodologia para essa construção e definir a linguagem e/ou ambiente a ser usado.

\section{Classificação e critérios para a construção de ontologias}

Diferentes tipos de ontologias podem ser definidos, levando-se em consideração o nível de generalidade (Guarino, 1998): ontologia de nível superior, de domínio, de tarefas e de aplicação.

Uma ontologia de nível superior ou de topo descreve conceitos gerais que são independentes do domínio, representando conceitos de espaço, tempo, eventos, objetos etc. Uma ontologia de domínio ou de tarefas especializa conceitos da ontologia de topo, descrevendo termos relacionados com um domínio genérico ou tarefas e atividades genéricas. Por exemplo: medicina, informática, meteorologia (ontologias de domínio), 
diagnóstico, vendas (ontologias de tarefas). A ontologia de aplicação descreve conceitos de um domínio ou tarefa em particular, especializando-os o máximo possível.

Do ponto de vista da engenharia de ontologias, uma ontologia é um produto que deve ser projetado para propósitos específicos. Segundo Grubber (1993a), alguns critérios de projeto de uma ontologia devem ser observados para garantir o propósito do compartilhamento de conhecimento e a interoperabilidade entre programas, tais como clareza e objetividade na definição dos termos, coerência, extensibilidade, codificação mínima para independência de uma tecnologia particular de representação de conhecimento e compromisso ontológico mínimo.

\section{Linguagens e ambientes para a construção de ontologias}

Existem várias propostas de linguagens para descrever ontologias. As linguagens se baseiam em formalismos, sendo a maioria delas baseadas em cálculo de predicados. Idealmente, para se fazer uma especificação ao nível do conhecimento usa-se uma linguagem de representação de ontologias independente de tipos de dados ou linguagens de programação.

A OWL (Web Ontology Language) foi recomendada pelo W3C (World Wide Web Consortium) em 2004, sendo projetada para aplicações que necessitam processar informações. OWL é baseada em XML, RDF e RDF-Schema, oferecendo mecanismos para uma semântica formal.

Dentre as diversas ferramentas que auxiliam na construção da ontologia, destacase o ambiente Protegé (Noy e McGuiness, 2001). É uma ferramenta integrada que permite ao usuário construir uma ontologia de domínio ou uma base de conhecimento, além de ser uma plataforma de código aberto escrito em Java que pode ser estendida para uso de tabelas, diagramas, gráficos, animações, através de instalações de plugins.

\section{Ontologia do eJLPT}

Assim, dado o potencial de expressividade das ontologias aplicáveis a um domínio e contexto, é apresentada, a proposta de utilização de ontologias para o mapeamento das estratégias de adaptação para direcionar o aluno no seu processo de aprendizagem, descrevendo-as através de ontologias de domínio para o JLPT. Isso envolve conceituar e mapear a ementa do curso de língua japonesa necessária ao conteúdo, ao nível de proficiência exigida e às regras estabelecidas pelo JLPT. Desta forma, foram definidos os seguintes requisitos para a ontologia em desenvolvimento:

- Deve permitir a representação de problemas, enunciados formais e suas resoluções de acordo com o estabelecido para o JLPT:

- As ontologias devem possuir significado semântico sobre nível da prova do JLPT, tempo para resposta da prova, peso das questões por relevância, entre outros;

- Deve representar formalmente a ementa do curso da Língua japonesa dentro do escopo do JLPT. Alguns elementos são visíveis:

- As ementas do curso são compostas de unidades de aula e estas, se classificam de acordo com o tipo (contexto) de prova do eJLPT, possuindo um contexto principal. Os contextos possíveis, segundo o JLPT são ortografia e vocabulário, compreensão auditiva e compreensão de texto e gramática;

- As unidades de aula possuem um assunto e um sub-assunto, bem como algumas propriedades: a unidade de aula-anterior, unidade de aula- 
subseqüente, nível do JLPT (Nível 1 ao 4) e contextos relacionados. As unidades de aula podem pertencer a mais de um contexto (aplicação);

- Os exercícios estão associados a contextos e unidades de aula.

- Deve permitir a comparação dos efeitos contextuais resultantes durante a aprendizagem do aluno no ambiente eJLPT e a comparação do esforço necessário para processar uma suposição. Deve permitir que um agente possa direcionar o aluno à obtenção da relevância ótima em um dado contexto;

- Deve ser extensível mantendo a consistência do conhecimento existente. Definiu-se como escopo inicial da ontologia os conteúdos e regras atualmente exigidas pelos JLPT para brasileiros.

\section{Uma taxonomia do eJLPT}

O vocabulário de uma ontologia é o conjunto de todos os objetos que existem no mundo. Para o JLPT, a ementa do curso é formada por objetos denominados unidades de aula (cumprimento formal, alfabeto Katakana, e outros). As unidades de aula estão associadas a um contexto principal e pertencem a um nível específico do JLPT.

As aulas possuem uma seqüência de contextos a serem expandidos, especificados através da navegação nas unidades de aula anteriores e subseqüentes. Cada unidade de aula pertence a um assunto e possui um sub-assunto. A simulação da prova em forma de exercício está relacionada às unidades de aula envolvidas no contexto do JLPT selecionado pelo aluno. Desta forma, o eJLPT consegue atender aos objetivos direcionados do aluno, medir a relevância de contexto do aluno e direcionar para a relevância ótima através de um agente.

Um modelagem inicial dos objetos e conceitos básicos foi feito utilizando-se a ferramenta Protegé. A Figura 3 é a exportação da ontologia ora definida ao IHMC COE CmapTools, em forma de mapas conceituais, facilitando a visualização da hierarquia (relação isa (é um) que define a relação de classe/subclasse) de conceitos do domínio.

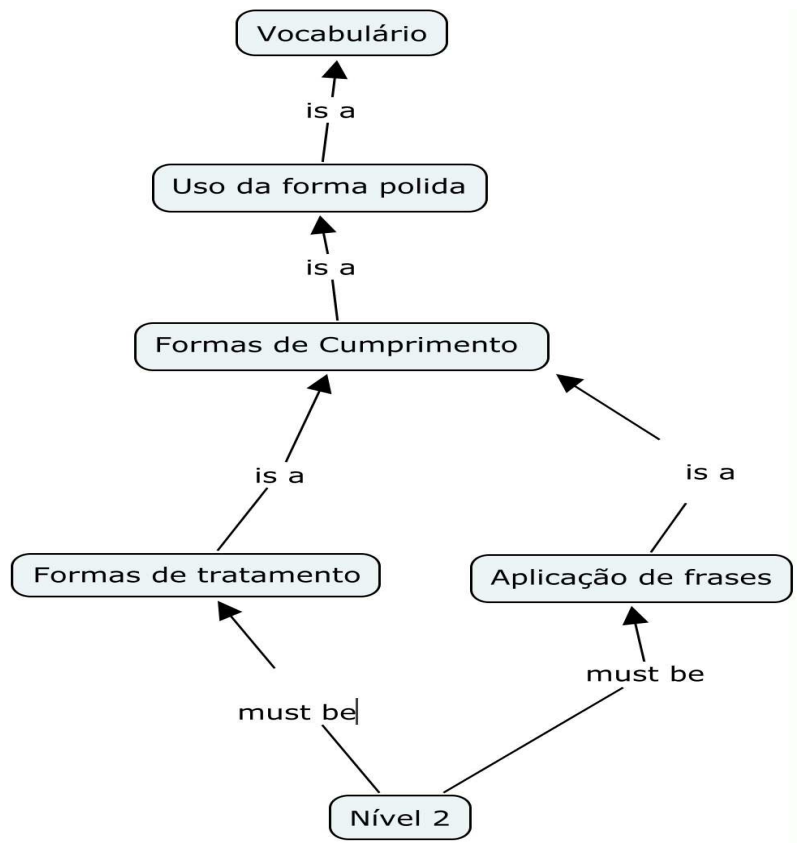

Figura 3 - Mapa conceitual de uma ontologia eJLPT (gerada no Protege e exportada para visualização no IHMC COE CmapTools) 


\section{PAT (Pedagogical and Affective Tutor)}

Os agentes pedagógicos animados usam recursos de multimídia para fornecer ao usuário um personagem animado com características semelhantes àquelas de seres vivos inteligentes. Essas características, tais como, expressões faciais e entendimento das emoções humanas, juntamente com uma boa interface de diálogo com o usuário, torna esses agentes mais atraentes ao aluno (Elliott e Brzezinski, 1998).

A agente a ser utilizada é a PAT (Jaques e Vicari, 2008), que aplica táticas pedagógicas afetivas que com o objetivo de promover um estado de espírito positivo no aluno, estimulando o aprendizado, bem como fornecendo um suporte emocional ao aluno, motivando-o e encorajando-o.

PAT reconhece as emoções satisfação/frustração, alegria/tristeza, gratidão/raiva e vergonha do aluno. As emoções do aluno são inferidas através das ações do aluno na interface do ambiente educacional. A inferência das emoções foi baseada no modelo OCC (Ortony, Clore e Collins, 1988), que é baseado na teoria cognitiva das emoções e é possível de ser implementado computacionalmente. Devido à natureza dinâmica da informação afetiva sobre o aluno, a abordagem BDI (Belief, Desire and Intention) foi adotada para o modelo afetivo do aluno.

\section{Considerações finais}

Foi apresentada neste trabalho a proposta de um esquema para se integrar um AVA em uma hipermídia adaptativa, bem como a sugestão de uma instanciação possível. Ao longo do trabalho, foram aprofundadas as discussões pertinentes a cada componente instanciado do esquema, de tal forma que se visualizasse a factibilidade de cada elemento isoladamente. Isto é suficiente para sustentar a próxima etapa do trabalho, que consiste na integração destes componentes em uma hipermídia adaptativa e validar o esquema proposto.

Uma das questões envolvidas na pesquisa é se o esquema proposto atende a integração de outros ambientes hipermídias adaptativos específicos ou se novos elementos precisam ser acoplados ao esquema.

Neste trabalho também foram apresentados aspectos do projeto de uma ontologia para o JLPT com a finalidade de ter recursos automáticos inteligentes adaptáveis aos anseios relacionados ao processo de aprendizagem de alunos interessados na certificação JLPT. Foram apresentados alguns requisitos que esta ontologia deve possuir para se adequar ao ambiente proposto e garantir o mapeamento dos dados e a obtenção da informação através de sistemas de computação.

Uma contribuição interessante deste trabalho está focada na decisão do uso de ontologias para ambientes de educação a distância. Neste caso específico, a grande contribuição surge para o ambiente eJLPT com a inserção de semântica nos documentos para que os agentes possam raciocinar sobre os mesmos, possibilitando ganhos no processo e nas preferências de aprendizagem do aluno, de tal forma que mantém o mesmo focado em contextos relevantes ao JLPT. Não se limitando ao ambiente, por outro lado, a modelagem e as estratégias adotadas permitem o reuso das ontologias, podendo ser aproveitado e compartilhado em outros ambientes da Web. 


\section{Referências bibliográficas}

BORST, W.N. Construction of engineering ontologies for Knowledge sharing and reuse. Thesis. University of Twenty, Enschede, Netherlands - Centre for Telematics and Information Technology, 1991, 243p.

BROWN, T.E. Transtorno de déficit de atenção: a mente desfocada em crianças e adultos. Portot Alegre: Artmed, 2007.

ELLIOTT, C. e BRZEZINSKI, J. Autonomous Agents as Synthetic Characters. AI Magazine, [S. 1.], v. 19, n. 2, 1998, p.13-30.

GASPARINI, I. Concepção de Interfaces WWW Adaptativas para EAD. Cadernos de Informática. Porto Alegre, v.2, n.1, 2002, p.71-76.

GUARINO, N. Formal ontology and information systems. Proceedings of the First International Conference on Ontology in Information Systems - FOIS'98. Trento, Italy, 1998, p.3-15.

JAQUES, P.A. e VICARI, R.M. Infering Emotions and Applying Affective Tactics for a Better Learning. In: R. M. Vicari, P. A. Jaques, et al (Ed.). Agent-Based Tutoring Systems by Cognitive and Affective Modeling. New York: IGI Global, 2008. Infering Emotions and Applying Affective Tactics for a Better Learning, 2008, p.135-155.

NOY, N.F. e MCGUINESS, D. Ontology Development 101: A Guide to Creating Your First Ontology. SMI Technical Report SMI-2001-0880, Stanford University, March, 2001.

ORTONY, A., CLORE, G. e COLLINS, A. The cognitive structure of emotions. Cambridge, UK: Cambridge University Press, 1988.

SIMULADOR e-JLPT. Disponível em: <www.ejlpt.web.br.com> Acesso em: 22 jul. 2005.

SPERBER, D. e WILSON, D. Relevance. Communication and Cognition. Oxford: Blackwell, 1995. 